\title{
Comparing Newborn Outcomes After Prenatal Exposure to Individual Antidepressants Claire Marks ${ }^{1}$, Sara Quinney ${ }^{2}$, David Haas ${ }^{2}$ \\ ${ }^{1}$ Indiana University School of Medicine; ${ }^{2}$ Indiana University School of Medicine, Department of Obstetrics and Gynecology
}

Background: Up to 1 out of 5 women experience depression during their childbearing years, with $20-33 \%$ treating with drugs. However, data are limited on individual drugs' effects on pregnancy outcomes. The objective of this study was to compare associations between individual antidepressants and pregnancy outcomes.

Study Methods: Deidentified demographic, medical and obstetric data from women who received at least one antidepressant (SSRI or SNRI) prescription prior to conception through delivery were obtained from Regenstrief Institute. Drugs were were compared using standard statistics and multivariable regression.

Results: A total of 3801 women took bupropion $(n=311)$, citalopram $(n=355)$, fluoxetine $(n=520)$, sertraline $(n=1557)$, duloxetine $(n=112)$, escitalopram $(n=534)$, venlafaxine $(n=105)$, paroxetine $(n=45)$, desvenlafaxine $(n=12)$, or multiple antidepressants $(n=250)$. Most women were white $(86.5 \%)$, and had either commercial $(50.3 \%)$ or government $(48.1 \%)$ insurance.

Race $(p=0.002)$, hospital $(p<0.001)$, and insurance $(p<0.001)$ were associated with selection of drug. Incidence of diabetes $(p<0.001)$, gestational diabetes $(p=0.005)$, hypertension $(p=0.015)$, and gestational hypertension $(p=0.006)$ varied between drugs, with highest rates in women taking duloxetine. NICU admission ( $p<0.001)$, transient tachypnea of newborn (TTN) $(p=0.001)$, and adaptation syndrome $(p<0.001)$ were significantly different between drugs, with duloxetine having highest NICU admission (42.9\%) and TTN (20.5\%). Paroxetine was associated with higher rates of adaptation syndrome (13.3\%).

After controlling for maternal age, race, hospital, and insurance, adaptation syndrome was increased with citalopram $\mathrm{OR}[95 \% \mathrm{Cl}]=2.358[1.147-4.849]$, duloxetine $3.180[1.362-7.424]$, escitalopram 2.832[1.418], paroxetine 3.347[1.138-9.847], and multiple drugs 2.603[1.2225.545] compared to bupropion. Adding hypertension and diabetes to the model above, increased NICU admission rates were seen with citalopram 1.498[1.036-2.168], fluoxetine 1.565[1.109-2.208], duloxetine 2.568[1.593-4.139], escitalopram 2.045[1.458-2.869], and multiple drugs 2.009[1.362-2.964] compared to bupropion.

Conclusion: Different antidepressants have associations with individual maternal and newborn outcomes. Duloxetine and paroxetine appear to have the strongest associations with NICU admission and adaptation syndromes, respectively. These outcomes require further investigation by exposure trimester. 